\title{
GENETICAL CONSEQUENCES OF SINGLE SEED DESCENT IN THE BREEDING OF SELF-POLLINATING CROPS
}

\author{
J. W. SNAPE and T. J. RIGGS \\ Plant Breeding Institute, Maris Lane, Trumpington, Cambridge CB2 2LQ
}

Received 27.i.75

\section{SUMmary}

\begin{abstract}
The theoretical changes in the mean and variance of a segregating population under single seed descent, a method of rapid generation advancement in self-pollinating crops, are discussed. The direction and magnitude of these changes are shown to depend on the genetical architecture of the character under consideration.

Examples, using computer simulation, show that for situations where heterosis is exhibited, few homozygous lines will be produced which exceed the mean of the $\mathrm{F}_{2}$ distribution from which they were derived.

The advantages of the method over the normal pedigree breeding method are discussed.
\end{abstract}

\section{INTRODUCTION}

IN breeding self-pollinating crops the objectives are to obtain true-breeding lines from hybrid progenies, and to select lines that have the desired combination of characters.

Goulden (1939) suggested that the segregating generations derived from hybridisation could be rapidly advanced in the greenhouse with no selection and taking only one or two progeny from each plant in each successive generation. Large numbers of near-homozygous lines can then be tested in the field only a short time after the initial hybridisation. This method is known as the modified pedigree method (Brim, 1966) or simply as single seed descent.

Application of the method to oat breeding has been discussed by Kaufmann (1961, 1971) and to wheat breeding by Knott and Kumar (1975). Theoretical work by Pesek and Baker (1969) has compared tandem and index selection for the modified pedigree method, and Baker (1971) has discussed the theoretical variance of response to selection in the $F_{n}$ generation derived by single seed descent. There has been little discussion, however, about the genetical consequences of the method on quantitative characters.

The present paper is concerned with examining the theoretical consequences of single seed descent for a character determined by different genetical architectures.

The techniques of computer simulation were used to provide simple examples.

\section{THEORY}

The expected means and variances of the single seed descent generations derived from a cross between two inbred lines are easily derived. 
The $F_{n}$ population will consist of a proportion $\left(\frac{1}{2}\right)^{n-1}$ individuals heterozygous for each segregating locus and a proportion $\frac{1}{2}\left[1-\left(\frac{1}{2}\right)^{n-1}\right]$ of each of the corresponding homozygotes. Then, after Mather and Jinks (1971) whose notation is used here, the mean, in the absence of epistasis will be

$$
\bar{F}_{n}=m+\left(\frac{1}{2}\right)^{n-1}[h]
$$

where $m$ is the midparent value and $[h]$ the balanced sum of the individual dominance effects.

If epistasis is present, this becomes

$$
\bar{F}_{n}=m+\left(\frac{1}{2}\right)^{n-1}[h]+\left(\frac{1}{2}\right)^{2 n-2}[l],
$$

where $m$ is now Van der Veen's $F \infty$ metric and $[l]$ the balanced sum of the individual heterozygote $\times$ heterozygote interactions.

The above equations, of course, simply give the generation means of a selfing series as given by Mather and Jinks (1971).

It can be seen that in the presence of dominance the mean expression of any character may change relative to the mean of the $F_{2}$, the direction and magnitude of the change depending on the direction and magnitude of the dominance effects, and if present, the epistatic effects at all loci.

Turning to the generation variances, the variance between individuals in the absence of interaction is

$$
V_{F_{n}}=\frac{2^{(n-1)}-1}{2^{(n-1)}} \cdot D+\frac{2^{(n-1)}-1}{2^{(2 n-2)}} \cdot H+E,
$$

where $D=S\left(d^{2}\right), H=S\left(h^{2}\right)$ and $E=$ environmental variance.

When epistatic effects are present, following Mather $(1967,1974)$ this becomes

$$
\begin{aligned}
\frac{2^{(n-1)}-1}{2^{(n-1)}} \cdot D+\frac{2^{(n-1)}-1}{2^{(2 n-2)}} \cdot H+\frac{\left(2^{(n-1)}-1\right)^{2}}{\left(2^{(n-1)}\right)^{2}} \cdot I+\frac{\left(2^{(n-1)}-1\right)^{2}}{2^{(3 n-3)}} \cdot J \\
\quad+\frac{\left(2^{(n-1)}-1\right)^{2}}{\left(2^{(2 n-2)}\right)^{2}} \cdot L+E
\end{aligned}
$$

where $I=S\left(i^{2}\right), J=S\left(j^{2}\right)$ and $L=S\left(l^{2}\right)$. that

Here, however, the definitions of $D$ and $H$ change over generations such

$$
\begin{aligned}
& D_{n}=S_{a}\left(d_{a}+\left(\frac{1}{2}\right)^{n-1} \cdot S_{b}\left(j_{a b}\right)\right)^{2} \\
& H_{n}=S_{a}\left(h_{a}+\left(\frac{1}{2}\right)^{n-1} \cdot S_{b} l\left({ }_{a b}\right)\right)^{2} .
\end{aligned}
$$

From these equations it can be seen that in the absence of interaction the variance of the population increases regardless of the presence or direction of dominance. This is to be expected due to the fixation of additive variance. However, in the presence of epistasis this is not necessarily so and changes in the variance will depend on the type and magnitudes of the epistatic components.

In the final generation, family structure is imposed so that the variance can be partitioned into between and within line components. 
The variance between the means of the near-homozygous lines becomes

$$
V_{1} F_{n}=\frac{2^{(n-2)}-1}{2^{(n-2)}} \cdot D+\frac{2^{(n-2)}-1}{2^{(2 n-2)}} \cdot H+E_{b}
$$

and the average variance within families

$$
V_{2} F_{n}=\frac{1}{2^{(n-1)}} \cdot D+\frac{1}{2^{n}} \cdot H+E_{w}
$$

excluding, for simplicity, the epistatic components.

\section{Examples}

A diploid, self-fertilising organism was simulated with two alleles at each of 21 loci of equal effect determining a single quantitative trait. Independent assortment of genes was allowed and the two parents exhibited maximum gene dispersal.

Four genetical situations were simulated:

(i) additive effects with complete dominance for increasing alleles;

(ii) additive effects only, i.e. no dominance;

(iii) additive effects with complete dominance for increasing alleles and complementary gene interactions;

(iv) additive effects with complete dominance for increasing alleles and duplicate gene interactions.

Genotype values were calculated using $m=22.5, d=0.5$ and $h=0.5$ for situations of complete dominance. For the simulation of epistasis 10 gene pairs were allowed to interact and epistasis was complete, i.e. $\theta=1$ (Mather, 1967). Complementary interaction was simulated by setting $h=i=j=l$ and duplicate interaction by setting $h=-i=-j=-l$, for each interacting pair.

Segregation in the progeny of the $F_{1}$ was simulated and $1500 F_{2}$ individuals generated. A single progeny was then produced from each $\mathrm{F}_{2}$ individual and three generations of single seed descent practised. In the final generation $\left(F_{6}\right)$ families were produced from each $F_{5}$ individual and family means and variances computed.

Figure 1 shows the observed genotypic means for successive generations of single seed descent for the different genetical architectures. It is easily shown that these are in very good agreement with their theoretical expectations.

Under all systems where dominance is present and is directional for the increasing alleles, a fall in the generation mean will occur. If there is no dominance or if dominance is ambidirectional, little change will occur other than by sampling.

The populations simulated exhibited heterosis in the $F_{1}$ and subsequent generations because a system of dispersed genes and directional dominance was assumed. Such crosses are likely to be of most interest to the plant breeder, particularly for yield. However, under single seed descent, as under normal selfing without selection, the mean expression of heterosis will regress towards the midparental value. 
Turning to the changes in genotypic variance (fig. 2), it can be seen that under all the genetical systems, in the absence of epistasis, the variance will increase from the $F_{2}$ to $F_{6}$ generations, although as homozygosity is approached sampling may cause the variance to fall slightly. Similarly when duplicate gene interactions are involved in the control of the character, the variance will increase. In contrast to this, complementary gene interactions cause the variance to fall in successive generations.

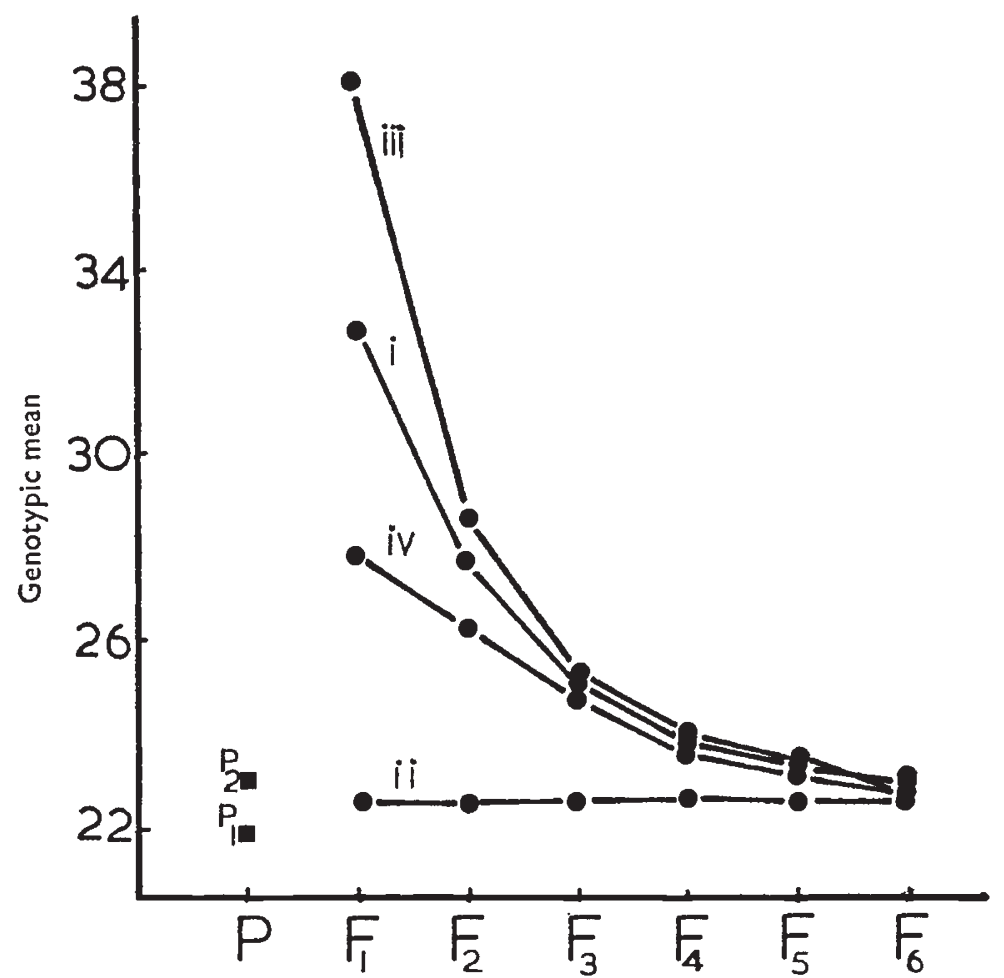

Frg. 1.-Changes in the genotypic mean over generations of single seed descent for genetical situations (i) to (iv) - - see text.

Of particular interest to the plant breeder using the single seed descent method are the distributions of the means of the $F_{6}$ lines compared to the distributions of the $\mathrm{F}_{2}$ individuals from which they were derived.

Figure $3 a$ and $b$ shows the distributions of $1500 \mathrm{~F}_{2}$ individuals and the $F_{6}$ lines derived from them for systems of complete dominance and no dominance with no epistasis. In the absence of dominance, the distribution of the means of $F_{6}$ lines is similar to the genotypic distribution of the $F_{2}$ individuals. Thus the transgressive segregation exhibited in the $F_{2}$ can be fixed in homozygous lines. None of the lines produced is as good as the extreme homozygote containing all the increasing alleles, but due to the increased variance lines better than the best $\mathrm{F}_{2}$ 's are produced.

When a large amount of directional dominance is exhibited, however, the $F_{6}$ distribution falls markedly away from that of the $F_{2}$ individuals and in the present example only about 2 per cent of the $F_{6}$ lines are better 
than the mean of the $F_{2}$ individuals from which they were derived. Again, however, transgressive segregation can be fixed.

The distributions obtained when epistasis and complete dominance are present are shown in fig. $4 a$ and $b$ for complementary and duplicate interactions respectively.

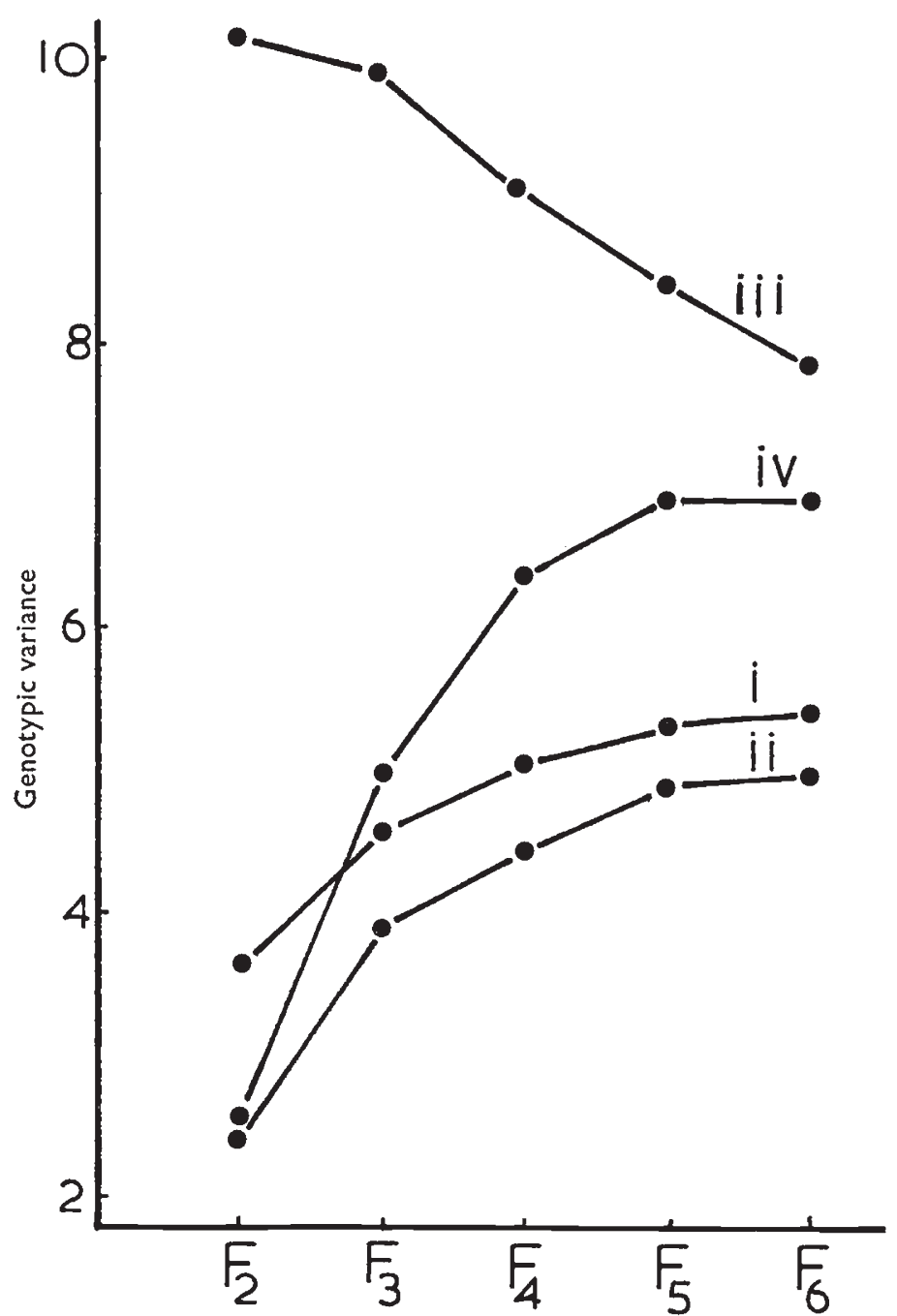

Fig. 2.-Changes in the genotypic variance over generations for genetical situations (i) to (iv).

Complementary interactions inflate the $F_{2}$ mean and variance. In subsequent generations, however, both mean and variance fall and few $\mathrm{F}_{6}$ lines achieve an expression as high as the mean of the $F_{2}$ population. This result is, therefore, very similar to the case of complete dominance with no interaction. In the presence of duplicate gene interactions nearly 40 per cent of the $F_{2}$ individuals achieve the extreme expression and the distribution is markedly skewed. The $F_{6}$ distribution, however, is much 

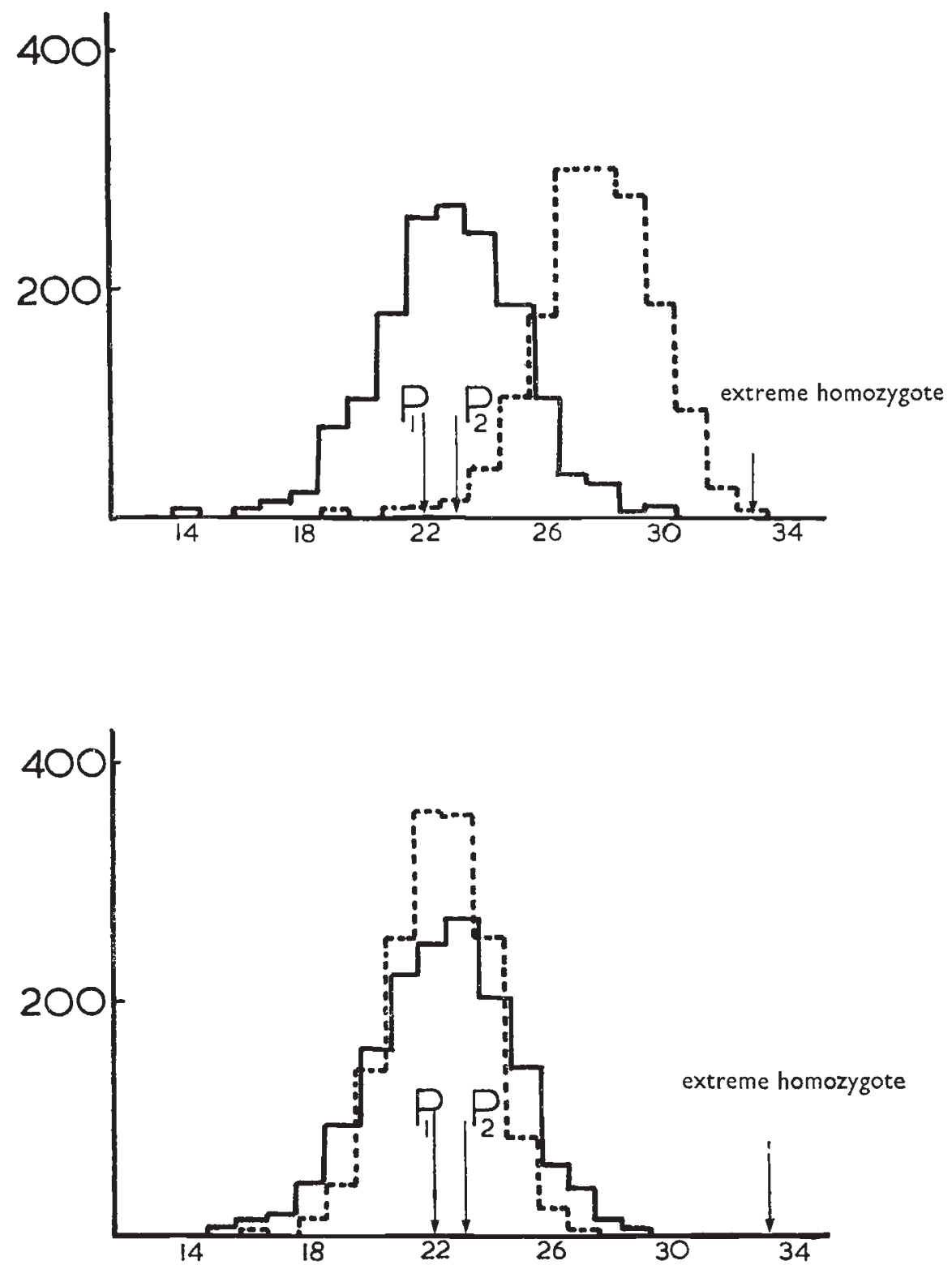

FIG. $3 a$ and $b .-$ Distributions of $F_{2}$ individuals $(--)$ and their $F_{6}$ lines $(-)$ for situations of complete dominance and no dominance, respectively.

less skewed and is very similar to the situation with no dominance and no interaction. Nevertheless, here 6 per cent of the $F_{6}$ lines achieve the extreme expression. For this type of genetical architecture, therefore, the same genetical advance could be obtained using single seed descent as with the normal pedigree method with selection in each generation.

Finally it will be noticed from figs. 3 and 4 that the distributions of the means of the $\mathrm{F}_{6}$ lines are similar, regardless of the genetical architecture 

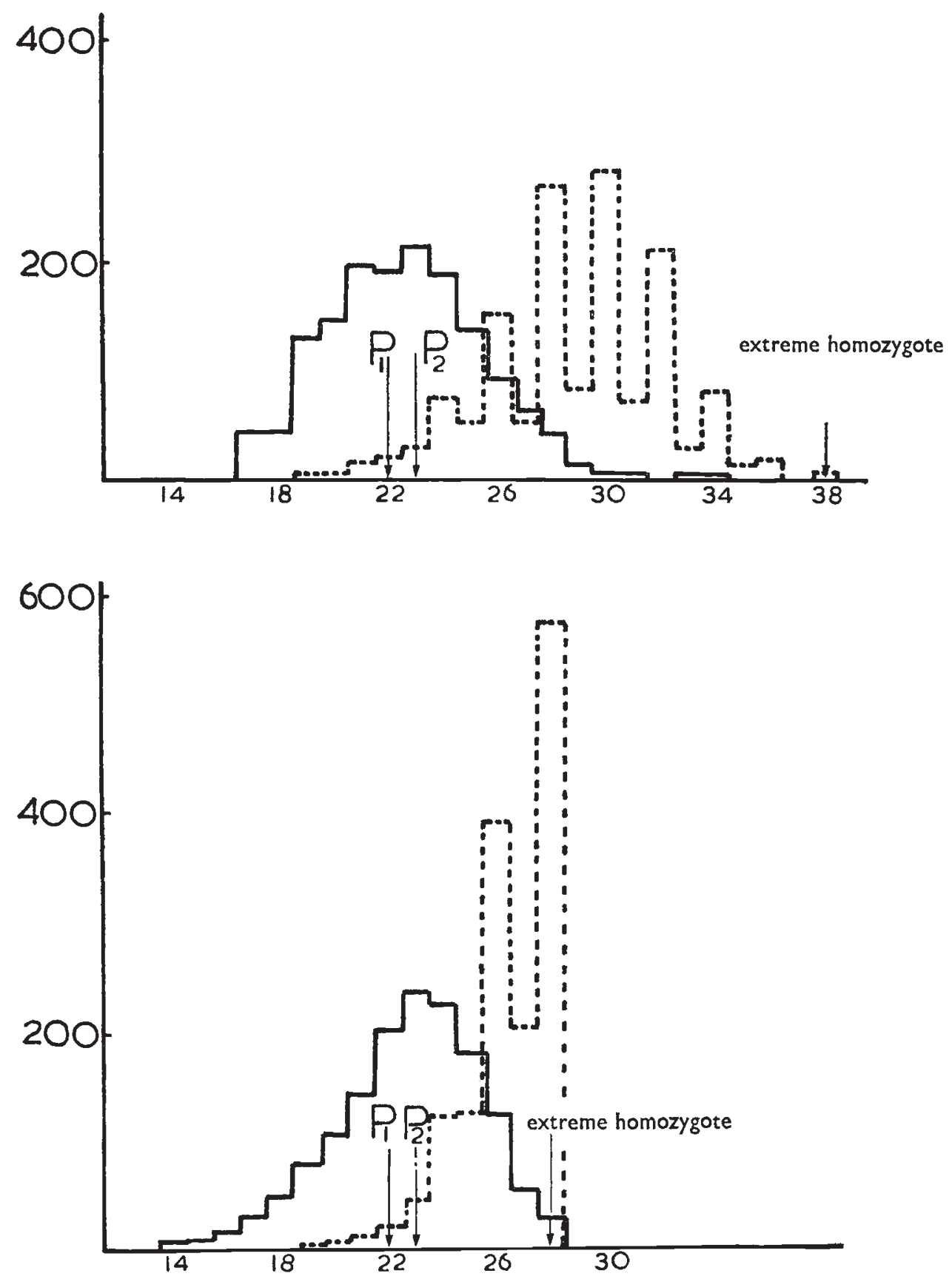

Frg. $4 a$ and $b$. - Distributions of $\mathrm{F}_{2}$ individuals $(--)$ and their $\mathrm{F}_{6}$ lines $(-)$ for situations of complementary and duplicate type gene interactions, respectively. 
assumed for the character, though the $\mathrm{F}_{2}$ distributions are very different. The most extreme lines in each distribution have almost the same phenotypes, but these phenotypes fall below the most extreme homozygote possible, except for the situation of duplicate gene interactions. Indeed, it is easily shown that for the other types of genetical architecture a very large population would have to be produced in order to secure such a line.

\section{Discussion}

The results of the present study illustrate that the distributions of $F_{6}$ lines after three generations of single seed descent will be similar whatever the genetical architecture of the character. Assessment of an $F_{2}$ population, however, will depend a great deal on the very different distributions resulting from different genetical situations. For situations where heterosis is exhibited, notably when systems of dispersed dominant genes and complementary gene interactions are displayed, the population of $F_{6}$ lines obtained will fall well below the expectations of the $F_{2}$, and a greater genetic advance would be expected by using the normal pedigree method.

In view of this result the advantages of the method over the normal pedigree selection method must be considered if it is to have widespread usage.

Firstly, selection in each generation from $F_{2}$ through to $F_{6}$ is costly in terms of both time and labour. Furthermore, whilst selection for highly heritable characters of agronomic importance may be effective, there is some doubt amongst breeders as to the effectiveness of early generation selection for yield. McGinnis and Shebeski (1968) reported that visual identification of high-yielding $\mathrm{F}_{2}$ plants by three selectors was successful. However, the correlations between $F_{2}$ plant yields and $F_{3}$ plot yields in the following season were not significant. This result was confirmed by De Pauw and Shebeski (1973). The efficiency of visual selection amongst wheat lines has been questioned also by Townley-Smith, Hurd and McBean (1973). Indeed, careful experimentation, costly in time and labour, is needed to identify high-yielding lines both within and over seasons in the segregating material. An important source of error will be the occurrence of genotype-environment interaction over sites and seasons in the segregating material. These may be of particular significance in view of the current trend of growing spring cereals in alternate hemispheres and thus obtaining two generations in a year.

Secondly, selection for agronomic and seed characters may be practised in the $\mathrm{F}_{2}$ for both the pedigree selection method and the single seed descent method. However, it has been pointed out by Kaufmann (1971) that because of negative correlations between several of these characters and yield, selection in the segregating generations could result in the rejection of high-yielding lines before they are recognised as such. Under a single seed descent procedure no selection is practised, except perhaps in the $\mathrm{F}_{2}$, and combinations of characters will be produced which might not have been selected but which nevertheless may be high yielding and of potential value as breeding stocks.

In the light of the above discussion, the single seed descent procedure may be seen as having some validity in plant breeding practice, and although genetic advance may not be maximised, transgressive segregation 
can be fixed and genetic advance obtained. Indeed, it has already been shown (Knott and Kumar, 1975) that lines obtained using the single seed descent procedure can be as good as those produced by the normal pedigree method. Clearly the savings in time and effort which the method offers should make it attractive to many plant breeders.

\section{ReFERENCES}

BAKER, R. J. 1971. Theoretical variance of response to modified pedigree selection. Can. 7. Plant Sci., 51, 463-468.

BRIM, C. A. 1966. A modified pedigree method of selection in soybeans. Crop Sci., 6, 220. DE PAUW, R. M., AND SHEBESKI, L. H. 1973. An evaluation of an early generation yield testing procedure in Triticum aestivum. Can. F. Plant Sci., 53, 465-470.

goulden, c. H. 1939. Problems in plant selection. In Proceedings of the Seventh International Genetics Congress. Cambridge University Press, pp. 132-133.

кaufmann, м. L. 1961. A proposed method of oat breeding for Central Alberta. Cereal News, 6(2), 15-18.

KaUfmanN, M. L. 1971. The random method of oat breeding for productivity. Can. $\mathcal{F}$. Plant Sci., 51, 13-16.

KNOTT, D. R., AND KUMAR, J. 1975. A comparison of early generation yield testing and a single seed descent procedure in wheat breeding. Crop Sci. 15, 295-299.

MATHER, K. 1967. Complementary and duplicate gene interactions in biometrical genetics. Heredity, 22, 97-103.

MATHER, K. 1974. Non-allelic interaction in continuous variation of randomly breeding populations. Heredity, 32, 414-419.

MATHER, K., AND JINKS, J. L. 1971. Biometrical Genetics. Chapman and Hall Ltd., London. MCGINNIS, R. C., AND SHEBESKI, L. H. 1968. The reliability of single plant selection for yield in $\mathrm{F}_{2}$. Proc. Third Intern. Wheat Symp., 410-415.

PESEK, J., AND BAKER, R. J. 1969. Comparison of tandem and index selection in the modified pedigree method of breeding self-pollinating species. Can. F. Plant Sci., 49, 773-781.

TOWNLEY-SMITH, T. F., HURD, E. A., AND MCBEAN, D. s. 1973. Techniques of selection for yield in wheat. Proc. Fourth Intern. Wheat Genet. Symp., 605-609. 